\title{
MATHEMATICAL MODELING OF THE FILTRATION CONSOLIDATION OF WATER-SATURATED RANDOMLY INHOMOGENEOUS SOIL MASSES
}

\author{
V. V. Skopetskii ${ }^{\dagger}$ and L. V. Volokh
}

UDC 519.21:517.977

\begin{abstract}
The filtration consolidation of water-saturated randomly inhomogeneous soil masses is studied. The field of excess head in a soil mass with random inclusions is obtained in the case of random consolidation coefficients.
\end{abstract}

Keywords: filtration consolidation, consolidation coefficients, excess head, consolidation of soil mass.

The Darcy law is known to be the principal law of filtration in the classical theory of nonstationary filtration in a porous medium. This law assumes that the equilibrium between pressure gradient and velocity is attained instantaneously. However, equilibrium is actually attained with a delay and, to take this into account, velocity $v$ and pressure $p$ in the corresponding relations should be replaced with $v+\lambda_{v} \frac{d v}{d t}$ and $p+\lambda_{p} \frac{d p}{d t}$, respectively, where $\lambda_{v}>0$ is relaxation of velocity and $\lambda_{p}>0$ is relaxation of pressure. Once the pressure jump is removed instantaneously, the motion does not stop instantaneously but damps as $\exp \left(-t / \lambda_{v}\right)$. As the motion stops instantaneously, the pressure gradient damps in time as $\exp \left(-t / \lambda_{p}\right)$. Disequilibrium between the velocity of filtration and the pressure gradient can be explained by relaxation effects due to the inertia of fluid and the velocity as a function of pressure gradient or due to the complexity of structure and the properties of the randomly nonuniform medium.

Let a rectangular soil mass $\Omega=\{(x, y): 0 \leq x \leq l, 0 \leq y \leq a\}$ contain randomly arranged spherical inclusions. The filtration properties of the material of inclusions (domains $\Omega_{i}$ with boundaries $\Gamma_{i}(i=\overline{1, n})$ ) substantially differ from the filtration characteristics of the matrix of the bed where an inclusion is located (the domain $\Omega_{0}$ with boundary $\Gamma_{0}$ ), in particular, the consolidation coefficients $c_{v_{i}}$ for domains $\Omega_{i}$ substantially differ from the consolidation coefficients $c_{v_{0}}$ of the domain $\Omega_{0}$.

Let us consider a problem similar to that from [1].

The consolidation equation in view of the creep of the soil skeleton is as follows:

$$
\tau_{1} \frac{\partial^{2} H}{\partial t^{2}}+\frac{\partial H}{\partial t}=c_{v_{0}}\left(\Delta H+\tau_{2} \frac{\partial}{\partial t}(\Delta H)\right)
$$

for the matrix of the bed and

$$
\tau_{1} \frac{\partial^{2} H}{\partial t^{2}}+\frac{\partial H}{\partial t}=c_{v_{1}}\left(\Delta H+\tau_{2} \frac{\partial}{\partial t}(\Delta H)\right), i=\overline{1, n},
$$

for inclusions, where $c_{v_{i}}$ is a random consolidation coefficient, $H$ is excess head, $\tau_{1}$ and $\tau_{2}$ are real parameters, and $\Delta=\frac{\partial^{2}}{\partial x^{2}}+\frac{\partial^{2}}{\partial y^{2}}$ is Laplacian.

Conditions at the interface between inclusion and the matrix can be written as

$$
\left.H\right|_{\vec{r} \in \Gamma_{i}-0}=\left.H\right|_{\vec{r} \in \Gamma_{i}+0},\left.\quad k_{i} \frac{\partial H}{\partial n}\right|_{\vec{r} \in \Gamma_{i}-0}=\left.k_{i+1} \frac{\partial H}{\partial n}\right|_{\vec{r} \in \Gamma_{i}+0},
$$

where $n$ is the normal to $\Gamma_{i}$.

V. M. Glushkov Institute of Cybernetics, National Academy of Sciences of Ukraine, Kyiv, Ukraine, †fiotkpi@public.icyb.kiev.ua. Translated from Kibernetika i Sistemnyi Analiz, No. 1, pp. 89-100, January-February 2008. Original article submitted May 25, 2007. 


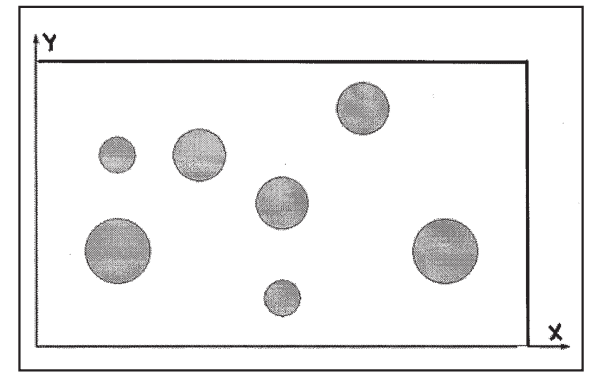

Fig. 1

Figure 1 shows a possible structure of a body with migrating impurity.

Let us write the dimensionless variables and parameters:

$$
x^{\prime}=\frac{x}{l}, y^{\prime}=\frac{y}{l}, t^{\prime}=\frac{t}{T}, H^{\prime}=\frac{H}{H_{0}}
$$

as

$$
\tau_{1}^{\prime}=\frac{\tau}{T}, c_{v}^{\prime}=\frac{c_{v} T}{l^{2}}, a^{\prime}=\frac{a}{l} \quad\left(i=1,2, H_{0}, T=\text { const }\right) .
$$

Passing in (1) and (2) to the dimensionless variables (4) and omitting the prime, we get the same form of equations as in (1) and (2). Let us write the boundary conditions:

$$
\begin{gathered}
H(0, y, t)=0, H(1, y, t)=0, \\
H(x, 0, t)=0, H_{y}^{\prime}(x, a, t)=0, \\
H(x, y, 0)=H_{0}(x, y), \\
H_{t}^{\prime}(x, y, 0)=0 .
\end{gathered}
$$

It is necessary to find the excess head field $H$ in the soil mass with randomly located inclusions provided that $c_{v}=c_{v}(\vec{r})$ is a random function.

Let us reduce the consolidation problem for a mass (1), (2), (5)-(8) to the problem for the whole body. To this end, we use the main derivations from [2]. Since the function $H$ within the body has discontinuities of the first kind, we use the representation

$$
f^{\prime}(x)=\left\{f^{\prime}(x)\right\}+\sum_{i}[f]_{x_{i}} \delta\left(x-x_{i}\right),
$$

where $\left\{f^{\prime}(x)\right\}$ is a piecewise-continuous function, $[f]_{x_{i}}$ is a discontinuity of the function $f(x)$ at the point $x_{i}$, and $\delta$ is the Dirac delta function.

For $H$,

$$
H^{\prime}=\left\{H^{\prime}\right\}+\sum_{i}[H]_{\Gamma} \delta\left(\vec{r}-\vec{r}_{\Gamma}\right)
$$

$\vec{r}=(x, y)$ is a position vector and $\vec{r}_{\Gamma}$ is a position vector of points of the boundary $\Gamma$ of the body.

Applying (9) repeatedly, we similarly find $\Delta H$. We get

$$
\Delta H(x, y, t)=\Delta H(\vec{r}, t)=\{\Delta H(\vec{r}, t)\}+[\nabla H(\vec{r}, t)]_{\Gamma} \delta\left(\vec{r}-\vec{r}_{\Gamma}\right)+[H(\vec{r}, t)]_{\Gamma} \nabla \delta\left(\vec{r}-\vec{r}_{\Gamma}\right),
$$

where $\Delta H=\frac{\partial^{2} H}{\partial x^{2}}+\frac{\partial^{2} H}{\partial y^{2}}, \nabla H=\frac{\partial H}{\partial x}+\frac{\partial H}{\partial y}$.

Let us introduce a random operator $\eta_{i}$ in terms of the Heaviside unit functions:

$$
\eta_{i}=\left\{\begin{array}{l}
1, \vec{r} \in \Omega_{i}, \\
0, \vec{r} \notin \Omega_{i},
\end{array} \quad \sum_{i=1}^{N} \eta_{i}(\vec{r})=1 .\right.
$$


Then the consolidation coefficient $c_{v}$ for the whole body can be written as $c_{v}(\vec{r})=\sum_{i=1}^{N} c_{v_{i}}(\vec{r}) \eta_{i}(\vec{r})$. Taking this into account, with (10) and the creep property, we will write the consolidation equation as

$$
\begin{gathered}
L(\vec{r}, t) H(\vec{r}, t)=\tau_{1} \frac{\partial^{2} H}{\partial t^{2}}+\frac{\partial H}{\partial t}-\sum_{i=1}^{N} c_{v_{i}}(\vec{r}) \eta_{i}(\vec{r}) \\
\times\left\{\{\Delta H(\vec{r}, t)\}+\sum_{i=1}^{N}[\nabla H(\vec{r}, t)]_{\Gamma_{i}} \delta\left(\vec{r}-\vec{r}_{\Gamma_{i}}\right)+\sum_{i=1}^{N}[H(\vec{r}, t)]_{\Gamma_{i}} \nabla \delta\left(\vec{r}-\vec{r}_{\Gamma_{i}}\right)\right. \\
\left.+\tau_{2} \frac{\partial}{\partial t}\left[\{\Delta H(\vec{r}, t)\}+\sum_{i=1}^{N}[\nabla H(\vec{r}, t)]_{\Gamma_{i}} \delta\left(\vec{r}-\vec{r}_{\Gamma_{i}}\right)+\sum_{i=1}^{N}[H(\vec{r}, t)]_{\Gamma_{i}} \nabla \delta\left(\vec{r}-\vec{r}_{\Gamma_{i}}\right)\right]\right\}=0 .
\end{gathered}
$$

Adding and subtracting in Eq. (11) a determinate operator $L_{0}(\vec{r}, t)$ defined on the interval $t \in[0, T], x \in[0,1]$, $y \in[0, a], L_{0}(\vec{r}, t)=\tau_{1} \frac{\partial^{2}}{\partial t^{2}}+\frac{\partial}{\partial t}-c_{v_{0}}\left(\Delta+\tau_{2} \frac{\partial}{\partial t}(\Delta)\right)$, yields

$$
L_{0}(\vec{r}, t) H(\vec{r}, t)=L_{i}(\vec{r}, t) H(\vec{r}, t),
$$

where

$$
\begin{gathered}
L_{i}(\vec{r}, t)=L_{0}-L=\left(c_{1}-c_{0}\right) \sum_{i=1}^{N} \eta_{i}(\vec{r}) \times\left\{\{\Delta\}+\sum_{i=1}^{N}[\nabla]_{\Gamma_{i}} \delta\left(\vec{r}-\vec{r}_{\Gamma_{i}}\right)\right. \\
\left.+\sum_{i=1}^{N}[\cdot]_{\Gamma_{i}} \nabla \delta\left(\vec{r}-\vec{r}_{\Gamma_{i}}\right)+\tau_{2} \frac{\partial}{\partial t}\left[\{\Delta\}+\sum_{i=1}^{N}[\nabla]_{\Gamma_{i}} \delta\left(\vec{r}-\vec{r}_{\Gamma_{i}}\right)+\sum_{i=1}^{N}[\cdot]_{\Gamma_{i}} \nabla \delta\left(\vec{r}-\vec{r}_{\Gamma_{i}}\right)\right]\right\} .
\end{gathered}
$$

Assume that the right-hand side of Eq. (12) is a source. The boundary-value problem (12), (5)-(8) is equivalent to the nonlinear integro-differential equation

$$
H(\vec{r}, t)=H_{0}(\vec{r}, t)=\int_{0}^{t} \int_{\Omega} G\left(\vec{r}, \vec{r}^{\prime}, t, t^{\prime}\right) L_{i}^{0}(\vec{r}, t) H(\vec{r}, t) d r d t,
$$

where $H_{0}(\vec{r}, t)$ is a solution of the homogeneous equation $L_{0} H_{0}=0$ with the boundary conditions (5)-(8).

Let us consider a homogeneous equation $L_{0}(\vec{r}, t) H(\vec{r}, t)=0$. Similarly to [4], to eliminate differential operations in $x$ and $y$, we solve it by the integral-transforms method.

Putting $M_{1} H=\frac{\partial^{2} H_{0}}{\partial x^{2}}$, we get the boundary-value problem for the kernel $\bar{K}$ :

$$
\frac{\partial^{2} \bar{K}}{\partial x^{2}}+\lambda^{2} \bar{K}=0,\left.\bar{K}\right|_{x=0}=\left.\bar{K}\right|_{x=1}=0
$$

The normalized solution of this problem is the function $\bar{K}=\frac{1}{\pi} \sin \lambda_{n} x, \lambda_{n}=n \pi$.

Then the equation $L_{0}(\vec{r}, t) H(\vec{r}, t)=0$ becomes

$$
\begin{gathered}
\tau_{1} \frac{\partial^{2} \bar{H}_{0}}{\partial t^{2}}+\frac{\partial \bar{H}_{0}}{\partial t}-c_{0}\left(\frac{\partial^{2} \bar{H}_{0}}{\partial y^{2}}-\lambda_{n}^{2} \bar{H}_{0}+\tau_{2} \frac{\partial}{\partial t}\left(\frac{\partial^{2} \bar{H}_{0}}{\partial y^{2}}-\lambda_{n}^{2} \bar{H}_{0}\right)\right)=0, \\
\left.\bar{H}_{0}\right|_{y=0}=0,\left.\frac{\partial \bar{H}_{0}}{\partial y}\right|_{y=a}=0, \\
\left.\bar{H}_{0}\right|_{t=0}=\bar{H}_{0}\left(\lambda_{n}, y\right), \bar{H}_{0}\left(\lambda_{n}, y\right)=\int_{0}^{1} H_{0}(x, y) \sin \lambda_{n} x d x,\left.\quad \frac{\partial \bar{H}_{0}}{\partial t}\right|_{t=0}=0 .
\end{gathered}
$$

To eliminate the differential operations in $y$, put $M_{2} H_{0}=\frac{\partial^{2} H_{0}}{\partial y^{2}}$. 
We obtain a problem similar to (14), with the kernel $\widetilde{K}=\frac{1}{\pi} \sin \mu_{m} x, \mu_{m}=\frac{\pi m}{a}, m \geq 1$.

Problem (15) becomes

$$
\begin{gathered}
\tau_{1} \frac{\partial^{2} \widetilde{H}_{0}}{\partial t^{2}}+\left(1+c_{0} \tau_{2}\left(\mu_{m}^{2}+\lambda_{n}^{2}\right)\right) \frac{\partial \widetilde{H}_{0}}{\partial t}+c_{0}\left(\mu_{m}^{2}+\lambda_{n}^{2}\right) \widetilde{H}_{0}=0, \\
\left.\widetilde{H}_{0}\right|_{t=0}=\widetilde{H}_{0}\left(\lambda_{n}, \mu_{m}\right), \\
\widetilde{H}_{0}\left(\lambda_{n}, \mu_{m}\right)=\int_{0}^{1} \int_{0}^{a} H_{0}(x, y) \sin \lambda_{n} x \sin \mu_{m} y d x d y=\int_{0}^{1} \int_{0}^{a} H_{0}(x, y) \sin \pi n x \sin \frac{\pi m}{a} y d x d y,\left.\quad \frac{\partial \widetilde{H}_{0}}{\partial t}\right|_{t=0}=0 .
\end{gathered}
$$

Thus, applying the integral Fourier transforms twice, we reduce problem (13) to the ordinary differential equation (16). Let $\widetilde{H}_{m n}^{0}(t)$ be a solution of this equation. Using the general expression for the inverse transform $u=\int_{a}^{b} u K(\lambda, \mu) \rho(\lambda) d \lambda$, we represent the solution $\bar{H}_{m n}^{0}(y, t)$ of problem (15) as a series

$$
\bar{H}_{n}^{0}(y, t)=\sum_{m=1}^{\infty} \widetilde{H}_{0}^{m n}(t) \widetilde{K}(y, m)=\sum_{m=1}^{\infty} \widetilde{H}_{0}^{m n}(t) \sin \frac{\pi}{a} m y .
$$

Applying the inverse transform once again yields the solution of the original problem (14)

$$
\begin{gathered}
H_{0}(x, y, t)=\sum_{n=1}^{\infty} \bar{H}_{0}^{n}(t) \bar{K}(x, n)=\sum_{n, m=1}^{\infty} \widetilde{H}_{0}^{m n}(t) \bar{K}(x, n) \widetilde{K}(y, m) \\
=\sum_{m=1}^{\infty} \widetilde{H}_{0}^{m n}(t) \sin \pi n x \sin \frac{\pi}{a} m y,
\end{gathered}
$$

where $\widetilde{H}_{0}^{m n}$ is a solution of problem (16) of the form

$$
\begin{gathered}
\widetilde{H}_{0}^{m n}=\frac{\widetilde{H}_{0}}{2}\left[\left(1+\frac{1+\tau_{2} K}{\sqrt{\left(1+\tau_{2} K\right)^{2}-4 \tau_{1} K}}\right) \exp \left\{\frac{t}{2 \tau_{1}}\left(\sqrt{\left(1+\tau_{2} K\right)^{2}-4 \tau_{1} K}-\left(1+\tau_{2} K\right)\right\}\right]\right. \\
+\left(1-\frac{1+\tau_{2} K}{\sqrt{\left(1+\tau_{2} K\right)^{2}-4 \tau_{1} K}}\right) \exp \left\{-\frac{t}{2 \tau_{1}}\left(\sqrt{\left(1+\tau_{2} K\right)^{2}-4 \tau_{1} K}+\left(1+\tau_{2} K\right)\right\}\right],
\end{gathered}
$$

where $K=C_{0}\left(\lambda_{n}^{2}+\mu_{m}^{2}\right)$.

Note that when $H_{0}(x, y)=H_{0}=$ const,

$$
\widetilde{H}_{0}=\left\{\begin{array}{l}
\frac{4 a H_{0}}{\pi^{2} n m}, n, m=2 k+1, \\
0, n, m=2 k
\end{array}\right.
$$

Let us introduce the following notation:

$$
\begin{aligned}
& \sqrt{\left(1+\tau_{2} K\right)^{2}-4 \tau_{1} K}+\left(1+\tau_{2} K\right):=A\left(\lambda_{n}, \mu_{m}\right), \\
& \sqrt{\left(1+\tau_{2} K\right)^{2}-4 \tau_{1} K}-\left(1+\tau_{2} K\right):=B\left(\lambda_{n}, \mu_{m}\right) .
\end{aligned}
$$

Then (17) becomes

$$
H_{0}(x, y, t)=\frac{1}{\pi^{2}} \sum_{n, m=1}^{\infty}\left[\frac{4 H_{0} a}{A\left(\lambda_{n}, \mu_{m}\right)+B\left(\lambda_{n}, \mu_{m}\right)}\right.
$$




$$
\times\left\{A\left(\lambda_{n}, \mu_{m}\right) \exp \left\{B\left(\lambda_{n}, \mu_{m}\right) \frac{t}{2 \tau_{1}}\right\}+B\left(\lambda_{n}, \mu_{m}\right) \exp \left\{-A\left(\lambda_{n}, \mu_{m}\right) \frac{t}{2 \tau_{1}}\right\}\right\} \times \sin \pi n x \sin \frac{\pi m}{a} y .
$$

Denote by $G(r, t)$ the Green function satisfying the equation for a point source:

$$
\tau_{1} \frac{\partial^{2} G(r, t)}{\partial t^{2}}+\frac{\partial G(r, t)}{\partial t}=c_{0}\left(\Delta G(r, t)+\tau_{2} \frac{\partial}{\partial t}(\Delta G(r, t))\right)=\delta\left(r-r^{\prime}\right)
$$

and the boundary conditions

$$
\begin{gathered}
\left.G(r, t)\right|_{t=0}=H_{0},\left.G_{t}^{\prime}(r, t)\right|_{t=0}=0, \\
\left.G(r, t)\right|_{r=0}=\left.G(r, t)\right|_{x=1}=0,\left.G_{y}^{\prime}(r, t)\right|_{y=a}=0 .
\end{gathered}
$$

Problem (20) can also be solved by the integral-transform method, similarly to problem (13).

Note that the Fourier transform of the function $\delta\left(r-r^{\prime}\right)$ has the form

$$
\begin{gathered}
\int_{0}^{1} \int_{0}^{a} \delta\left(x-x^{\prime}\right) \delta\left(y-y^{\prime}\right) \sin \pi n x \sin \frac{\pi m}{a} y d y d x \\
=\int_{0}^{1}\left[\int_{0}^{a} \delta\left(y-y^{\prime}\right) \sin \frac{\pi m}{a} y d y\right] \delta\left(x-x^{\prime}\right) \sin \pi n x d x=\sin \pi n x^{\prime} \sin \frac{\pi m}{a} y^{\prime}=0, \quad(n, m) \in N .
\end{gathered}
$$

We obtain the equation

$$
\tau_{1} \frac{\partial^{2} \widetilde{G}}{\partial t^{2}}+\left(1+c_{0} \tau_{2}\left(\xi_{m}^{2}+\psi_{n}^{2}\right)\right) \frac{\partial \widetilde{G}}{\partial t}+c_{0}\left(\zeta_{m}^{2}+\psi_{n}^{2}\right) \widetilde{G}=\sin \zeta_{n} x^{\prime} \sin \psi_{m} y^{\prime}=\tilde{\delta}_{m n} .
$$

Denoting $P=c_{0}\left(\zeta_{m}^{2}+\psi_{n}^{2}\right)$ and accounting for the boundary conditions of problem (20), we write the solution of this equation as

$$
\begin{gathered}
\widetilde{G}_{n m}=\frac{\tilde{H}_{0}-\tilde{\delta}_{m n} / P}{2 \sqrt{\left(1+\tau_{2} P\right)^{2}-4 \tau_{1} P}} \\
\times\left[\left(\sqrt{\left(1+\tau_{2} P\right)^{2}-4 \tau_{1} P}+\left(1+\tau_{2} P\right)\right) \exp \left\{\frac{t}{2 \tau_{1}}\left(\sqrt{\left(1+\tau_{2} P\right)^{2}-4 \tau_{1} P}-\left(1+\tau_{2} P\right)\right)\right\}\right. \\
\left.+\left(\sqrt{\left(1+\tau_{2} P\right)^{2}-4 \tau_{1} P}\left(1+\tau_{2} P\right)\right) \exp \left\{-\frac{t}{2 \tau_{1}}\left(\sqrt{\left(1+\tau_{2} P\right)^{2}-4 \tau_{1} P}+\left(1+\tau_{2} P\right)\right)\right\}\right]+\frac{\tilde{\delta}_{m n}}{p} .
\end{gathered}
$$

Let us introduce the notation similarly to (19):

$$
\begin{aligned}
& \sqrt{\left(1+\tau_{2} P\right)^{2}-4 \tau_{1} P}+\left(1+\tau_{2} P\right):=D\left(\xi_{n}, \psi_{m}\right), \\
& \sqrt{\left(1+\tau_{2} P\right)^{2}-4 \tau_{1} P}-\left(1+\tau_{2} P\right):=E\left(\xi_{n}, \psi_{m}\right),
\end{aligned}
$$

then

$$
\begin{gathered}
G(r, t)=\sum_{m, n=1}^{\infty} \widetilde{G}_{m n}(t) \sin \pi n x \sin \frac{\pi m}{a} y=\sum_{m, n=1}^{\infty}\left\{\frac{\tilde{H}_{0}-\tilde{\delta}_{m n} / P}{D+E}\right. \\
\left.\left.\times\left\{D\left(\xi_{n}, \psi_{m}\right) \exp \left\{E\left(\xi_{n}, \psi_{m}\right) \frac{t}{2 \tau_{1}}\right\}+E\left(\xi_{n}, \psi_{m}\right) \exp \left\{-D\left(\xi_{n}, \psi_{m}\right) \frac{t}{2 \tau_{1}}\right\}\right\}\right]+\frac{\tilde{\delta}_{m n}}{P}\right\} \times \sin \pi n x \sin \frac{\pi m}{a} y .
\end{gathered}
$$

We solve the integro-differential equation (13) by the method of successive approximations. As zero approximation $H^{0}(\vec{r}, t)$, we take the solution of the homogeneous boundary-value problem $H^{0}(\vec{r}, t)=H_{0}$. We have the following recurrent formulas: 


$$
\begin{gathered}
H^{(1)}(\vec{r}, t)=H_{0}+\int_{0}^{t} \int_{\Omega} G\left(\vec{r}, \vec{r}^{\prime}, t\right) L_{i}\left(\vec{r}^{\prime}, t\right) H_{0}\left(\vec{r}^{\prime}, t\right) d r^{\prime} d t, \\
H^{(2)}(\vec{r}, t)=H_{0}+\int_{0}^{t} \int_{\Omega_{1}} \int_{\Omega} G\left(\vec{r}, \vec{r}^{\prime}, t\right) L_{i}\left(\vec{r}^{\prime}, t\right) H^{(1)}\left(\vec{r}^{\prime}, t\right) d r^{\prime} d t, \ldots, \\
H^{(n)}(\vec{r}, t)=H_{0}+\int_{0}^{t} \int_{\Omega_{1}} \int_{\Omega} G\left(\vec{r}, \vec{r}^{\prime}, t\right) L_{i}\left(\vec{r}^{\prime}, t\right) H^{(n-1)}\left(\vec{r}^{\prime}, t\right) d r^{\prime} d t .
\end{gathered}
$$

Since $H_{0}$ is a continuously differentiable function,

$$
L_{i} H_{0}(\vec{r}, t)=\left(c_{1}-c_{0}\right) \sum_{i=1}^{N} \eta_{i}(\vec{r}) \times\left[\left\{\Delta H_{0}\right\}+\tau_{2} \frac{\partial}{\partial t}\left\{\Delta H_{0}\right\}\right] .
$$

The general term of the sequence $H^{(0)}, H^{(1)}, \ldots, H^{(n)}$ can be written as

$$
\begin{gathered}
H^{(n)}=H_{0}+\int_{0}^{t} \int_{\Omega} G\left(\vec{r}, \vec{r}^{\prime}, t\right) L_{i}\left(\vec{r}^{\prime}\right) H_{0}\left(\vec{r}^{\prime}, t\right) d r^{\prime} d t \\
+\int_{0}^{t} \int_{\Omega} G\left(\vec{r}, \vec{r}^{\prime}, t\right) L_{i}\left(\vec{r}^{\prime}\right) \int_{0}^{t} \int_{\Omega^{\prime}} G\left(\vec{r}^{\prime}, \vec{r}^{\prime \prime}\right) L_{i}\left(\vec{r}^{\prime \prime}\right) H_{0}\left(\vec{r}^{\prime \prime}, t^{\prime \prime}\right) d r^{\prime \prime} d r^{\prime} d t \\
+\int_{0}^{t} \int_{\Omega} G\left(\vec{r}, \vec{r}^{\prime}, t\right) L_{i}\left(\vec{r}^{\prime}\right) \int_{0}^{t^{\prime}} \int_{\Omega^{\prime}} G\left(\vec{r}^{\prime}, \vec{r}^{\prime \prime}\right) L_{i}\left(\vec{r}^{\prime \prime}\right) \times \ldots \\
\times \int_{0}^{t^{(n-2)}} \int_{\Omega^{(n-2)}} G\left(\vec{r}^{(n-2)}, \vec{r}^{(n-1)}\right) L_{i}\left(\vec{r}^{(n-1)}, H_{0}\left(\vec{r}^{(n-1)}, t\right) d r^{(n-1)} \ldots d r^{\prime} d t+R_{n}(\vec{r}, t),\right.
\end{gathered}
$$

where $R_{n}(r, t)$ is the difference between the $n$th and the $(n-1)$ th terms of the sequence

$$
\begin{aligned}
& R_{n}=\int_{0}^{t} \int_{\Omega} G\left(\vec{r}, \vec{r}^{\prime}, t\right) L_{i}\left(\vec{r}^{\prime}\right) \int_{0}^{t} \int_{\Omega} G\left(\vec{r}^{\prime}, \vec{r}^{\prime \prime}, t\right) L_{i}\left(\vec{r}^{\prime \prime}\right) \ldots \\
& \left.\int_{0}^{t} \int_{\Omega} G\left(\vec{r}^{(n-1)}, \vec{r}^{(n)}, t\right) L_{i}\left(\vec{r}^{(n)}\right)\right) H_{0}\left(\vec{r}^{(n)}, t\right) d r^{(n)} d r^{\prime} d t .
\end{aligned}
$$

We get the series

$$
H(r, t)=H_{0}(r, t)+\sum_{n} R_{n}(r, t)
$$

THEOREM 1. Series (23) converges absolutely and continuously.

Proof.

$$
\begin{gathered}
\left|L_{i}(r) H_{0}(r, t)\right| \leq\left|c-c_{0}\right| \sum_{i}\left|\eta_{i}(\vec{r})\right|\left|\left\{\Delta H_{0}\right\}+\tau_{2} \frac{\partial}{\partial t}\left\{\Delta H_{0}\right\}\right| \\
\leq c_{*} \sum_{i}\left|\left\{\Delta H_{0}\right\}+\tau_{2} \frac{\partial}{\partial t}\left\{\Delta H_{0}\right\}\right|
\end{gathered}
$$

$\left\{\Delta H_{0}\right\}$ is a piecewise continuous function, $H_{0}=\sum_{m, n} \widetilde{H}_{0}^{m n}(t) \sin \pi n x \sin \frac{\pi m}{a} y$. Then

$$
\Delta H_{0}=\frac{\partial^{2} H_{0}}{\partial x^{2}}+\frac{\partial^{2} H_{0}}{\partial y^{2}}=-\sum_{m, n} \widetilde{H}_{0}^{m n}(t)\left(\pi^{2} n^{2}+\frac{\pi^{2} m^{2}}{a^{2}}\right) \sin \pi n x \sin \frac{\pi m}{a} y,
$$




$$
\frac{\partial}{\partial t} \Delta H_{0}=2 \sum_{m, n} \frac{c_{0} \widetilde{H}_{0}\left(\pi^{2} n^{2}+\frac{\pi^{2} m^{2}}{a^{2}}\right)}{A+B} \sin \pi n x \sin \frac{\pi m}{a} y \times\left\{\exp \left(\frac{B}{2 \tau_{1}} t\right)-\exp \left(-\frac{A}{2 \tau_{1}} t\right)\right\}
$$

whence

$$
\begin{aligned}
& \begin{aligned}
\left|\left\{\Delta H_{0}\right\}+\tau_{2} \frac{\partial}{\partial t}\left\{\Delta H_{0}\right\}\right| & \leq \sum_{m, n}\left[\frac{\widetilde{H}^{0}}{2}\left|\frac{1}{A+B}\left(A \exp \left(\frac{B}{2 \tau_{1}} t\right)+B \exp \left(-\frac{A}{2 \tau_{1}} t\right)\right)\left(\pi^{2} n^{2}+\pi^{2} \frac{m^{2}}{a^{2}}\right)\right|\right. \\
& +2 \tau_{2} C_{0} \sum_{m, n} \frac{\left(\pi^{2} n^{2}+\pi^{2} \frac{m^{2}}{a^{2}}\right) \widetilde{H}^{0}}{A+B}\left|\sin \pi n x \sin \frac{\pi m}{a}\right| \\
\leq \sum_{m, n}\left[\frac{2 a H^{0}}{\pi^{2} n m} \mid\right. & \left.\frac{1}{A+B}\left(A \exp \left(\frac{B}{2 \tau_{1}} t\right)+B \exp \left(-\frac{A}{2 \tau_{1}} t\right)\right)\left(\pi^{2} n^{2}+\pi^{2} \frac{m^{2}}{a^{2}}\right) \mid\right] \leq U=\text { const. } \\
& \left|R_{n}\right| \leq U \mid \int_{0}^{t} \int_{\Omega}^{t} G\left(\vec{r}, \vec{r}^{\prime}, t, t^{\prime}\right) \int_{0}^{t^{\prime}} \int_{\Omega} G\left(\vec{r}^{\prime}, \vec{r}^{\prime \prime}, t^{\prime}, t^{\prime \prime}\right) \times \ldots
\end{aligned}
\end{aligned}
$$

Therefore,$$
\times L_{i}\left(\vec{r}^{(n-1)}\right) \int_{0}^{t^{(n-1)}} \int_{\Omega} G\left(\vec{r}^{(n-1)}, \vec{r}^{(n)}, t^{(n-1)}, t^{(n)}\right) d r^{(n)} d t^{(n)} d r^{\prime} d t^{\prime} .
$$

The operator $L_{i}(\vec{r})$ acts on the Green function $G\left(r, r^{\prime}, t, t^{\prime}\right)$ as follows:

$$
\begin{gathered}
L_{i} G=\left(c-c_{0}\right) \sum_{i=1}^{n} \eta_{i}(\vec{r})\left\{\Delta G+\tau_{2} \frac{\partial}{\partial t}(\Delta G)\right\}, \\
\Delta G=-\sum_{m, n} \widetilde{G}_{m n}(t)\left(\pi^{2} n^{2}+\frac{\pi^{2} m^{2}}{a^{2}}\right) \sin \pi n x \sin \frac{\pi m}{a} y, \\
\frac{\partial \Delta G}{\partial t}=-\sum_{m, n} \frac{\partial}{\partial t} \widetilde{G}_{m n}(t)\left(\pi^{2} n^{2}+\frac{\pi^{2} m^{2}}{a^{2}}\right) \sin \pi n x \sin \frac{\pi m}{a} y \\
=2 \sum_{m, n} \frac{c_{0}\left(\pi^{2} n^{2}+\frac{\pi^{2} m^{2}}{a^{2}}\right) \widetilde{H}_{0}}{A+B} \sin \pi n x \sin \frac{\pi m}{a} y\left\{\exp \left(\frac{B}{2 \tau_{1}} t\right)-\exp \left(-\frac{A}{2 \tau_{1}} t\right)\right\} .
\end{gathered}
$$

Then

$$
\begin{gathered}
L_{i} G=\left(c-c_{0}\right) \sum_{i=1}^{n} \eta_{i}(\vec{r})\left[\sin \pi n x \sin \frac{\pi m}{a} y \sin \pi n x^{\prime} \sin \frac{\pi m}{a} y^{\prime}\right. \\
\left.+\sin \pi n x \sin \frac{\pi m}{a} y \times \frac{P \widetilde{H}_{0}-\sin \pi n x^{\prime} \sin \frac{\pi m}{a} y^{\prime}}{D+E} \times\left\{\left(D-2 P \tau_{2}\right) \exp \left(\frac{E}{2 \tau_{1}} t\right)+\left(E+2 P \tau_{2}\right) \exp \left(-\frac{D}{2 \tau_{1}} t\right)\right\}\right] .
\end{gathered}
$$

Since $\left|\eta_{i}(\vec{r})\right| \leq 1$ is the constraint for series

$$
\sum_{m, n} \sin \pi n x \sin \frac{\pi m}{a} y \sin \pi n x^{\prime} \sin \frac{\pi m}{a} y^{\prime} \exp \left(\frac{E}{2 \tau_{1}} t\right) \leq u_{1},
$$




$$
\sum_{m, n} \sin \pi n x \sin \frac{\pi m}{a} y \sin \pi n x^{\prime} \sin \frac{\pi m}{a} y^{\prime} \exp \left(-\frac{D}{2 \tau_{1}} t\right) \leq u_{2},
$$

we obtain

$$
\begin{aligned}
& \left|R_{n}\right| \leq U \mid \int_{0}^{t} \int_{\Omega} G\left(\vec{r}, \vec{r}^{\prime}, t\right) L_{S}\left(\vec{r}^{\prime}\right) \int_{0}^{t^{\prime}} \int_{\Omega} G\left(\vec{r}^{\prime}, \vec{r}^{\prime \prime}, t\right) L_{S}\left(\vec{r}^{\prime \prime}\right) \times \\
& \ldots \times L_{i}\left(\vec{r}^{(n-1)}\right) \int_{0}^{t} \int_{\Omega} G\left(\vec{r}^{(n-1)}, \vec{r}^{(n)}, t\right) d x^{(n)} d t^{(n)} \ldots d r^{\prime} d t^{\prime} \mid .
\end{aligned}
$$

As a result,

$$
\begin{gathered}
\left|R_{n}\right| \leq U \mid \int_{0}^{t} \int_{\Omega} G\left(\vec{r}, \vec{r}^{\prime}, t\right) L_{S}\left(\vec{r}^{\prime}\right) \int_{0}^{t} \int_{\Omega} G\left(\vec{r}^{\prime}, \vec{r}^{\prime \prime}, t\right) L_{S}\left(\vec{r}^{\prime \prime}\right) \times \ldots \\
\times \int_{0}^{t} \int_{\Omega}\left[\sin \pi n x^{(n)} \sin \frac{\pi m}{a} y^{(n)} \sin \pi n x^{(n-1)} \sin \frac{\pi m}{a} y^{(n-1)} \times \sin \pi n x^{(n)} \sin \frac{\pi m}{a} y^{(n)} \times \frac{P \widetilde{H}_{0}}{D+E}\right. \\
\left.\times\left\{\left(D-2 P \tau_{2}\right) \exp \left(\frac{E}{2 \tau_{1}} t\right)+\left(E+2 P \tau_{2}\right) \exp \left(-\frac{D}{2 \tau_{1}} t\right)\right\}\right] d x^{(n)} d y^{(n)} d t \mid .
\end{gathered}
$$

Since

$$
\int_{\Omega} \sin \pi n x \sin \frac{\pi m}{a} y d x d y=\frac{a^{2}}{\pi^{2} m^{2} n^{2}}\left((-1)^{n}-1\right)\left((-1)^{\frac{m}{a}}-1\right)
$$

and

we have

$$
\begin{aligned}
& \int_{0}^{1} \int_{\Omega} \exp \left(\frac{E}{2 \tau_{1}} t\right) \sin \pi n x \sin \frac{\pi m}{a} y d x d y d t \\
= & \frac{a^{2}}{\pi^{2} m^{2} n^{2}}\left((-1)^{n}-1\right)\left((-1)^{\frac{m}{a}}-1\right) \frac{2 \tau_{1}}{E} \exp \left(\frac{E}{2 \tau_{1}} t\right),
\end{aligned}
$$

$$
\begin{aligned}
\left|R_{n}\right| \leq U & \frac{a^{2}}{\pi^{2} n^{2} m^{2}} \mid \int_{0}^{t} \int_{\Omega} G\left(\vec{r}, \vec{r}^{\prime}, t\right) L_{S}\left(\vec{r}^{\prime}\right) \int_{0}^{t} \int_{\Omega} G\left(\vec{r}^{\prime}, \vec{r}^{\prime \prime}, t\right) L_{S}\left(\vec{r}^{\prime \prime}\right) \times \\
& \ldots \times L_{i}\left(\vec{r}^{(n-1)}\right) \int_{0}^{t^{(n-1)}} \int_{\Omega} G\left(\vec{r}^{(n-1),} \vec{r}^{(n)}, t\right) d_{r}{ }^{(n)} d t d r^{\prime} .
\end{aligned}
$$

Repeating this procedure $n-1$ times yields the estimate

$$
\begin{aligned}
& \left|R_{n}\right| \leq U\left|\left(\frac{a^{2}}{\pi^{2} m^{2} n^{2}}\right)^{(k-1)}\left((-1)^{n}-1\right)^{(k-1)}\left((-1)^{\frac{m}{a}}-1\right)^{(k-1)}\right| \\
& \times\left|\frac{P \tilde{H}_{0}}{D+E} \times\left\{\left(\frac{\left(D-2 P \tau_{2}\right) 2 \tau_{1}}{E}\right)^{(k-1)} \exp \left(\frac{E}{2 \tau_{1}} t\right)+\left(\frac{\left(E+2 P \tau_{2}\right) 2 \tau_{1}}{D}\right)^{(k-1)} \exp \left(-\frac{D}{2 \tau_{1}} t\right)\right\}\right| \\
& \leq C *\left(\frac{a}{\pi m n}\right)^{(k)} \times \frac{\tilde{P} \tilde{H}_{0}}{|D+E|} \times\left|\left(\frac{\left(D-2 P \tau_{2}\right) 2 \tau_{1}}{E}\right)^{(k-1)} u_{1}+\left(\frac{\left(E+2 P \tau_{2}\right) 2 \tau_{1}}{D}\right)^{(k-1)} u_{2}\right| .
\end{aligned}
$$


Since the series with the positive general term $C_{*}\left(\frac{a}{\pi m n}\right)^{(k)} \frac{P \widetilde{H}_{0}}{|D+E|} \mid\left(\frac{\left(D-2 P \tau_{2)} 2 \tau_{1}\right.}{E}\right)^{(k-1)} u_{1}+$ $\left(\frac{\left(E+2 P \tau_{2} 2 \tau_{1}\right.}{D}\right)^{(k-1)} u_{2} \mid$ converges as $n \rightarrow \infty$ for any values of $u_{1}, u_{2}, U, C_{*}$, and $t$, the sequence $\left\{H^{(n)}(\vec{r}, t)\right\}$ of partial sums of series (22) absolutely and uniformly converges as $n \rightarrow \infty$ according to the Weierstrass criterion, i.e., $\lim _{n \rightarrow \infty} H^{(n)}(\vec{r}, t)=H(\vec{r}, t)$.

Theorem 1 is proved.

THEOREM 2. The function $H(\vec{r}, t)=H_{0}(\vec{r}, t)+\sum_{n=1}^{\infty} R_{n}(\vec{r}, t)$ is a solution of the integro-differential equation (13).

The proof is similar to that in [2].

To find the excess head field $H$ in the mass with randomly located inclusions, we use the results from [3]. Let us restrict ourselves to the first two terms of series $(22)$, i.e., $H(\vec{r}, t) \approx H^{(1)}(\vec{r}, t)$. Let inclusions in the body be arranged uniformly with the distribution density $1 / S$ ( $S$ is the body area). Since $H_{0}(\vec{r}, t)$ is a nonrandom function, $\left\langle H_{0}(\vec{r}, t)\right\rangle_{\mathrm{exc}}=H_{0}(\vec{r}, t)$.

Consider the expression $I=\int_{0}^{t} \int_{\Omega} G\left(\vec{r}, \vec{r}^{\prime}, t, t^{\prime}\right) L_{i}\left(\vec{r}^{\prime}\right) H_{0}\left(\vec{r}^{\prime}, t\right) d r^{\prime} d t$. The random variables are position vectors of the centers of inclusions. Since

$$
\eta_{i}(\vec{r})=\left\{\begin{array}{c}
1, \vec{r} \in \Omega_{i} \\
0, \vec{r} \notin \Omega_{i}
\end{array}=\left\{\begin{array}{l}
1,\left|\vec{r}^{\prime}-\vec{r}_{i}\right| \in\left[0, R_{i}\right], \\
0,\left|\vec{r}^{\prime}-\vec{r}_{i}\right| \notin\left[0, R_{i}\right],
\end{array}=\eta_{i}\left(\left|\vec{r}^{\prime}-\vec{r}_{i}\right|\right)\right.\right.
$$

(where $\vec{r}_{i}$ is the position vector of the center of inclusion $\Omega_{i}$ and $R_{i}$ is the mean radius of inclusion),

$$
\langle I\rangle=\left(c_{1}-c_{0}\right) \int_{\Omega}\left(\left[\{\Delta H\}+\tau_{2} \frac{\partial}{\partial t}\left\{\Delta H_{0}\right\}\right] \frac{1}{S} \sum_{i=1}^{n_{1}} \int_{S} \eta_{i 1}\left(\vec{r}^{\prime}\right) d \vec{r}_{i 1}\right) d \vec{r}^{\prime} .
$$

Obviously,

$$
\frac{1}{S} \sum_{i=1}^{n_{1}} \int_{S} \eta_{i 1}\left(\vec{r}^{\prime}\right) d \vec{r}_{i 1}=\left\{\begin{array}{l}
\frac{1}{S} \sum_{i=1}^{n_{1}} \pi R_{1}^{2}=\frac{1}{2} s_{1}, r^{\prime} \in\left[0, R_{1}\right], \\
-\frac{1}{S} \sum_{i=1}^{n_{1}} \pi R_{1}^{2}=-\frac{1}{2} s_{1}, r^{\prime} \in\left[R_{1}, r_{0}-R_{1}\right] .
\end{array}\right.
$$

Then averaging yields

$$
\langle I\rangle=\frac{1}{2} s_{1}\left(c_{1}-c_{0}\right)\left\{\int_{0}^{R_{1}}\left(G \Delta H_{0}+\tau_{2} G \frac{\partial}{\partial t} \Delta H_{0}\right) d r^{\prime}-\int_{R_{1}}^{r_{0}-R_{1}}\left(G \Delta H_{0}+\tau_{2} G \frac{\partial}{\partial t} \Delta H_{0}\right) d r^{\prime}\right\},
$$

where

$$
\begin{gathered}
G \Delta H_{0}+\tau_{2} G \frac{\partial}{\partial t} \Delta H_{0}=\sum_{n, m=1}^{\infty}\left\{\frac{\widetilde{H}_{0}-\left(\sin \pi n x^{\prime} \sin \frac{\pi m}{a} y^{\prime}\right) / P}{A+B}\right. \\
\left.\times\left\{A \exp \left\{B \frac{t}{2 \tau_{1}}\right\}+B \exp \left\{-A \frac{t}{2 \tau_{1}}\right\}\right\}+\frac{\sin \pi n x^{\prime} \sin \frac{\pi m}{a} y^{\prime}}{P}\right\} \\
\times\left(\sin \pi n x \sin \frac{\pi m}{a} y\right)^{2} \times \frac{P}{c_{0}} \times \frac{\left(-\widetilde{H}_{0}\right)}{A+B} \times\left\{A \exp \left(\frac{B}{2 \tau_{1}} t\right)+B \exp \left(-\frac{A}{2 \tau_{1}} t\right)\right\}
\end{gathered}
$$




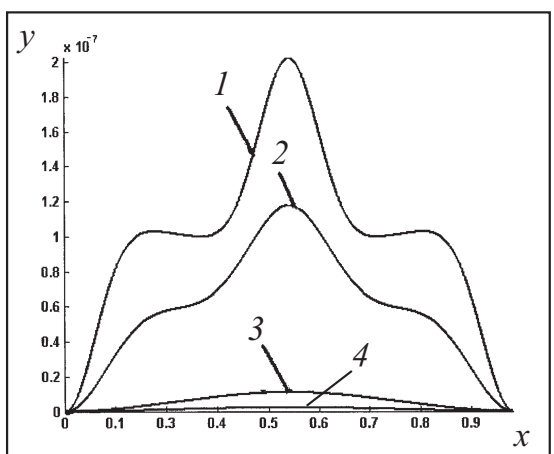

Fig. 2

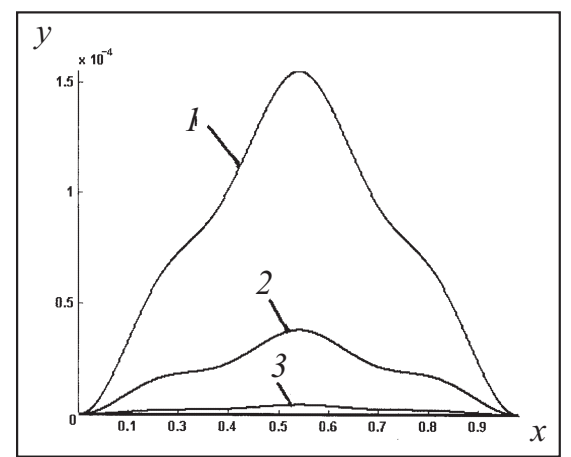

Fig. 3

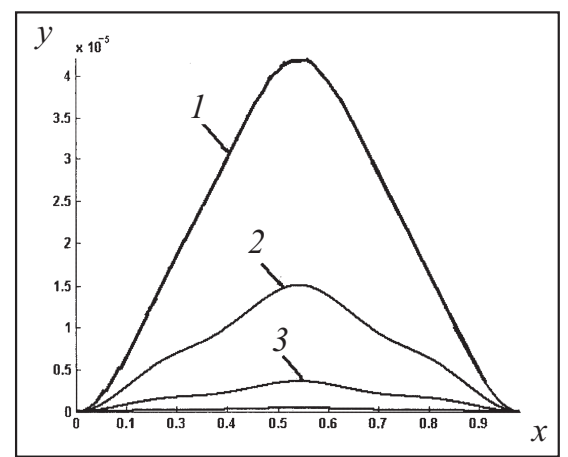

Fig. 4

$$
\begin{gathered}
\left.+2 \tau_{2} \sum_{n, m=1}^{\infty}\left\{\frac{\widetilde{H}_{0}-\left(\sin \pi n x^{\prime} \sin \frac{\pi m}{a} y^{\prime}\right) / P}{A+B} \times\left\{A \exp \left\{B \frac{t}{2 \tau_{1}}\right\}+B \exp \left\{-A \frac{t}{2 \tau_{1}}\right\}\right\}\right]+\frac{\sin \pi n x^{\prime} \sin \frac{\pi m}{a} y^{\prime}}{P}\right\} \\
\times\left(\sin \pi n x \sin \frac{\pi m}{a} y\right)^{2} \times \frac{P c_{0} \widetilde{H}_{0}}{A+B} \times\left\{\exp \left\{B \frac{t}{2 \tau_{1}}\right\}-\exp \left\{-A \frac{t}{2 \tau_{1}}\right\}\right\} .
\end{gathered}
$$

Substituting the Green function (22) and the excess head (17) into (24) and integrating yields the formula for the excess head field in a soil mass with randomly arranged inclusions provided that $c_{v}=c_{v}(\vec{r})$ is a random function.

For illustration, we will present the curves of the excess head field in a soil mass with randomly arranged inclusions.

Figure 2 shows the excess head field as a function of the ratios of consolidation coefficients $c_{v_{0}} / c_{v_{1}}=0.001$ (curves 1 and 2) and $c_{v_{0}} / c_{v_{i}}=0.01$ (curves 3 and 4 ) at the instants of time $t=10$ (curves 1 and 3 ), $t=20$ (curves 2 and 4 ) for $s_{1}=$ $3.14 \times 10^{-6}$ and $R_{1}=0.001$.

Figure 3 shows the excess head field for different values of the volumetric part of inclusions: $s_{1}=3.14 \times 10^{-6}$; $12.56 \times 10^{-6} ; 28.26 \times 10^{-6} ; 50.24 \times 10^{-6}$ for $c_{v_{0}} / c_{v_{i}}=0.001$ for $t=20$, curves $1-4$ corresponding to changes in $S_{1}$, curve 4 coinciding with the axis.

Figure 4 illustrates the influence of the typical radius of inclusions on the behavior of the excess head field, where $R_{1}=2 \times 10^{-2} ; 3 \times 10^{-3} ; 4 \times 10^{-3} ; 5 \times 10^{-3}$ correspond to curves $1-4$, curve 4 coinciding with the axis.

To solve the boundary-value problem of filtration consolidation of water-saturated randomly nonuniform soil masses, the following approach is proposed. The theory of distributions is used to reduce the boundary-value problem to a consolidation equation for the whole body, given the appropriate initial and boundary conditions. The boundary-value problem thus obtained is associated with a nonlinear integro-differential equation. The method of successive approximations is used to obtain the solution of this equation as an absolute and uniformly converging series. There is an excess head field in soil mass with randomly located inclusions provided that $c_{v}=c_{v}(\vec{r})$ is a random function. The present study is necessary to analyze filtration consolidation of drained soils with allowance for the geological properties of the soil skeleton.

\section{REFERENCES}

1. V. M. Bulavats'kyi, Yu. G. Krivonos, and V. V. Skopets'kyi, Nonclassical Mathematical Models of Heat and Mass Transfer [in Ukrainian], Naukova Dumka, Kyiv (2005).

2. O. Yu. Chernukha, "Describing diffusion processes in a ball with random spherical inclusions," Visnyk Lviv. Univ., Issue 1, 129-142 (2003).

3. I. V. Sergienko, V. V. Skopetskii, and V. S. Deineka, Mathematical Modeling and Analysis of Processes in Inhomogeneous Media [in Russian], Naukova Dumka, Kyiv (1991).

4. V. M. Bulavatskii and V. V. Skopetskii, "System approach to mathematical modeling of filtration consolidation," Cybern. Syst. Analysis, 42, No. 6, 831-838 (2006). 